FAMIIIAR chronic nail candidiasis (FCNC) is a rare disorder characterized by early-onset infections caused by different species of Candida, restricted to the nail of the hands and feet, and associated with a low serum concentration of intercellular adhesion molecule 1. Host defense mechanisms against candidiasis require the cooperation of many immune cells through several candidacidal mechanisms, including oxygen-dependent killing mechanisms, mediated by a superoxide anion radical myeloperoxidase $-\mathrm{H}_{2} \mathrm{O}_{2}-$ halide system, and reactive nitrogen intermediates. We analyzed protein carbonyl groups (considered a useful marker of oxidative stress) in the serum of patients belonging to a five-generation Italian family with an isolated form of FCNC.

Serum protein carbonyl groups in FCNC patients were significantly lower than those measured in healthy donors.

Also, if this hypothesis is merely speculative, we could suggest that the decreased circulating level of protein carbonyl groups in these patients is not a marker of a lower oxidative stress condition, but might be linked to a lower protease activity.

Key words: Chronic mucocutaneous candidiasis, Familiar chronic nail candidiasis, Protein carbonyl groups, Oxidative stress

\section{Protein carbonyl group content in patients affected by familiar chronic nail candidiasis}

\author{
S. Gangemi ${ }^{1}$, A. Saija ${ }^{2}$, A. Tomaino ${ }^{2}$, F. Cimino ${ }^{2}$, \\ R. A. Merendino ${ }^{3}$, P. L. Minciullo ${ }^{1}$, S. Briuglia ${ }^{4}$, M. V. \\ Merlino $^{4}$, B. Dallapiccola ${ }^{5}$, B. Ferlazzo ${ }^{1}$ and D. C. \\ Salpietro $^{4, C A}$
}

${ }^{1}$ Division and School of Allergy and Clinical Immunology, Department of Human Pathology, ${ }^{2}$ School of Pharmacy, Department of FarmacoBiologico, ${ }^{3}$ Chair of Immunopathology, Department of Human Pathology, and ${ }^{4}$ Paediatric Immunology and Genetics Operative Unit, University of Messina, Italy; ${ }^{5}$ RCCS-CSS San Giovanni Rotondo, CSSMendel, Rome, Italy

\author{
${ }^{\mathrm{CA}}$ Corresponding Author \\ Dipartimento di Scienze Pediatriche Mediche e \\ Chirurgiche, Policlinico Universitario, Padiglione NI, \\ 98125 Messina, Italy \\ Tel.: + 39902213114 \\ Fax: + 39906782336 \\ E-mail: sgangemi@eniware.it
}

Chronic mucocutaneous candidiasis (CMC) includes a group of rare disorders with altered immune responses, characterized by chronic infections of the skin, nails, and mucous membranes, caused by organisms of the genus Candida (mainly Candida albicans). Familial occurrence of CMC, first reported in $1972,{ }^{1}$ affects both males and females, and consanguinity is often present in pedigrees. The inheritance can be either autosomal dominant or recessive. Most CMC types are associated with altered phagocytosis and chemotaxis.

We previously described a distinct form of familiar chronic nail candidiasis (FCNC), characterized by early-onset infections caused by different species of Candida, restricted to the nail of the hands and feet, and associated with a low serum concentration of intercellular adhesion molecule-1 (ICAM-1). ${ }^{2}$ Afterwards, we carried out a genome-wide scan assigning the CMC locus to a $19 \mathrm{cM}$ pericentromeric region on chromosome $11 .^{3}$

It is generally agreed that oxidative stress, leading to accumulation of modified proteins (apart from damaged lipids), is involved in progression of several disorders and diseases. However, protein peroxidation, in contrast to lipid peroxidation, does not have the features of chain reactions.

The action of reactive oxygen species on proteins has been widely demonstrated to result in the formation of carbonyl groups. Carbonyl stress may be also due to toxic effects of various monodicarbonyls (such as malonyldialdehyde and 4-hydroxy-2,3-nonenal) and $\alpha$-dicarbonyls.

The level of these modified molecules can be quantified by measurement of the protein carbonyl content, which has been shown to increase in a variety of diseases and processes. Since the circulating proteins destructed by peroxidation have a quite long half-life period, the evaluation of carbonyl group content in blood proteins is considered a useful marker of oxidative stress.

In light of these findings, we analyzed the serum protein carbonyl content in seven patients belonging to a five-generation Italian family with an isolated form of CMC, affecting nails only, in the presence of a low serum concentration of ICAM-1, to clarify the oxidative stress in this disease. 
The study family originates from a rural village in Sicily and includes 11 affected subjects in seven generations. All affected family members presented nail dystrophy with hyperkeratosis and dark and thick nails. They were affected by onycomycosis caused by Candida involving all the nails of hands and feet. All patients had been treated over the years by topical and systemic antimycotic drugs, which resulted in temporary recovery, while never reaching complete remission.

All subjects underwent exhaustive tests excluding possible other systemic diseases.

After informed consent, a blood sample was collected from seven (three male, four female; mean age, 29.8 years; range, 5-48 years) affected family members, untreated with any systemic drugs at least 4 weeks prior to blood collection; 14 healthy donors, sex and age matched, were also enrolled as a control group.

Sera were obtained from peripheral blood allowed to clot at room temperature for $2 \mathrm{~h}$, separated by centrifugation at $200 \mathrm{x} g$ for $15 \mathrm{~min}$ in a $4235 \mathrm{~A}$ (ALC Int. S.r.L., Milan, Italy) centrifuge, and stored at $-80^{\circ} \mathrm{C}$ until use.

The carbonyl group serum content was evaluated with use of the Levine method. Briefly, $100 \mu \mathrm{l}$ of serum was incubated with $100 \mu \mathrm{l}$ of $20 \mathrm{mM} \mathrm{2,4-}$ dinitro-phenylhydrazine solution for $60 \mathrm{~min}$. Then the proteins were precipitated from the solution with the use of $20 \%$ trichloroacetate; the protein pellet was washed three times with ethanol and ethyl acetate, and re-suspended in $1 \mathrm{ml}$ of $6 \mathrm{M}$ guanidine at $37^{\circ} \mathrm{C}$ for $15 \mathrm{~min}$. The carbonyl content was determined from the absorbance at $366 \mathrm{~nm}$ (molar absorption coefficient, $22,000 / \mathrm{M} / \mathrm{cm}$ ). Differences in serum levels were assessed by one-way analysis of variance and the Student-Newman-Keults test. Data are expressed as the mean \pm standard deviation. $p<$ 0.05 was considered significant.

Our results show that serum levels of carbonyl proteins in FCNC patients were significantly lower than those measured in healthy donors (12.74 \pm 4.47 $\mathrm{nmol} / \mathrm{ml}$ versus $17.42 \pm 2.89 \mathrm{nmol} / \mathrm{ml}$ ) (Fig. 1). One has to point out that this is the first research carried out on serum protein carbonyl content in patients affected by CMC.

No correlation between disease activity/severity and the individual level of carbonyl groups of each patient was found.

C. albicans is an opportunistic yeast member of the normal microbial flora, inhabiting as a commensal mucous membrane $>80 \%$ of immunocompetent adults.

However, C. albicans becomes invasive and causes serious diseases in immunocompromised hosts (such as leukemic, diabetic, organ transplant, and human immunodeficiency virus-infected pa- tients) and in various non-immunological situations (i.e. systemic antibiotics, systemic steroid treatment).

Host defense mechanisms against candidiasis are complex and require the cooperation of many immune cells through several candidacidal mechanisms, including oxygen-dependent killing mechanisms, mediated by a superoxide anion radical myeloperoxidase $-\mathrm{H}_{2} \mathrm{O}_{2}$-halide system, and reactive nitrogen intermediates.

To elucidate the role of anti-oxidant systems in $C$. albicans, the ALO1 gene of $C$. albicans - which encodes the enzyme d-arabinono-1,4-lactone oxidase (ALO) that catalyzed the final step of d-erythroascorbic acid (a five-carbon analog of 1-ascorbic acid) biosynthesis - was disrupted by the URA blaster technique. ${ }^{4}$ The resulting alo1-alo1 null mutants showed increased $C$. albicans susceptibility to macrophage attack, reduced hyphal growth and attenuated virulence in mice.

Therefore, antioxidant defense systems are, for $C$. albicans, essential to its survivability and virulence.

FCNC is characterized by chronic infections caused by different species of Candida, restricted to the nails of the hands and feet with ICAM-1 deficiency, linked to incapacity of host of complete remission after topical and systemic antimycotic drugs.

An explanation of this incapacity may be represented by the low ICAM-1 serum levels detectable in these patients. In fact, ICAM-1 is a glycoprotein membrane and a member of the immunoglobulin superfamily, which plays a central role in cell to cellmediated immune response, and is a ligand for leucocyte function associated antigen-1. Genetically engineered mice, lacking ICAM-1 expression, had a significantly higher mortality rate following an intravenous challenge with $C$. albicans compared with normal wild mice. 5

We observed in ICAM-1-deficient patients circulating levels of carbonyl groups significantly lower than those measured in normal subjects. This is a surprising finding, because we have previously observed that serum malonyldialdehyde content in the same population is higher than that of controls (unpublished data).

One could suppose that a different regulation of ICAM-1-related biochemical pathyways (e.g., in the expression of protease inhibitors) may underlie the low levels of oxidized proteins observed in ICAM-1deficient patients. In fact, the destructed proteins are not repaired and are selectively removed by proteinases; several authors have shown that there is a relationship between protein oxidation and proteolytic activity, and changes in the efficiency of proteolysis can affect accumulation of oxidatively modified proteins. Furthermore oxidized and modified proteins are able to modulate the expression of adhesion molecules, such as ICAM-1, and the protease-anti-protease balance. 


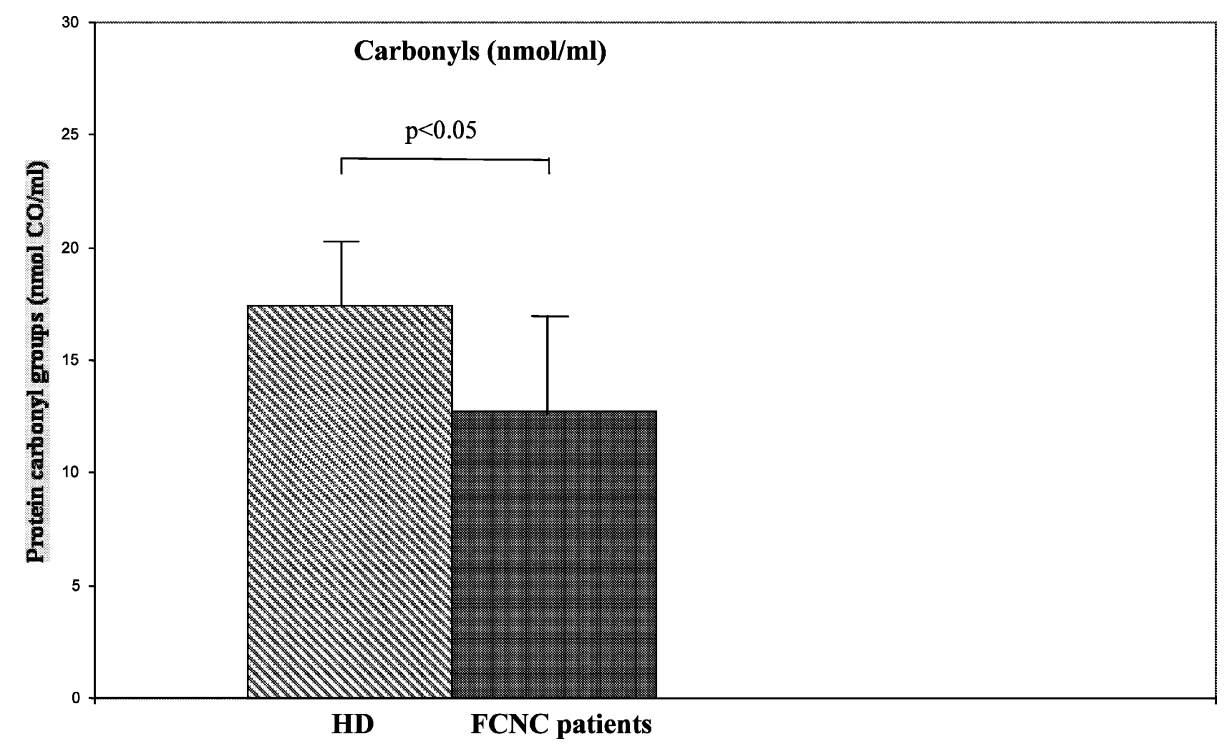

FIG. 1. Protein carbonyl groups serum levels in FCNC patients and healthy donors (HD). Data points are from individual measurements performed in triplicate.

Thus, we could suggest that the decreased circulating level of protein carbonyl groups in these patients is not a marker of a lower oxidative stress condition, but might be linked to a lower protease activity.

Of course this suggestive hypothesis is merely speculative, and thus further investigations are needed to establish the altered defense network in these subjects.

\section{References}

1. Wells RS, Higgs JM, McDonald A, Valdimarsson H, Holt PJL. Familial chronic muco-cutaneous candidiasis. J Med Genet 1972; 9: 302-310.
2. Zuccarello D, Salpietro DC, Gangemi S, et al. Familial chronic nail candidiasis with ICAM-1 deficiency: a new form of chronic mucocutaneous candidiasis. J Med Genet 2002; 39: 671-675.

3. Mangino M, Salpietro DC, Zuccarello D, et al. A gene for familial isolated chronic nail candidiasis (CMC) maps to chromosome 11p12-q12.1. Eur J Hum Genet 2003; 11: 433-436.

4. Huh WK, Kim ST, Kim H, Jeong G, Kang SO. Deficiency of ${ }_{d}$ Erythroascorbic acid attenuates hyphal growth and virulence of Candida albicans Infect Immun 2001; 69: 3939-3946.

5. Davis SL, Hawkins EP, Mason EO Jr, Smith CW, Kaplan SL. Host defences against disseminated candidiasis are impaired in intercellular adhesion molecule 1 deficient mice. J Infect Dis 1996; 174: 435-439.

\section{Received 25 April 2003 \\ Accepted 8 May 2003}




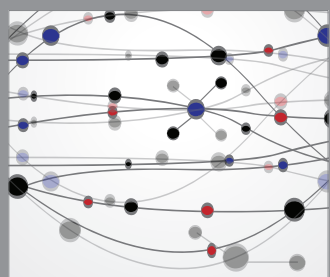

The Scientific World Journal
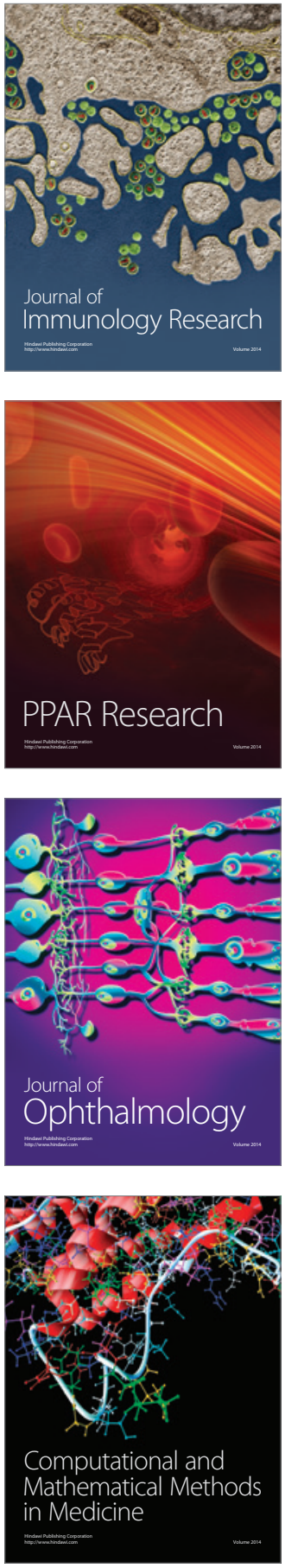

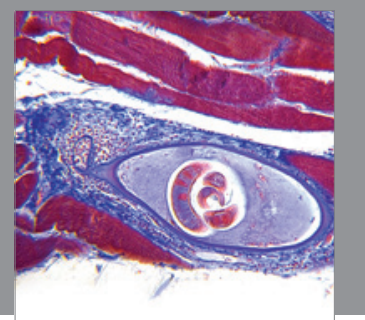

Gastroenterology

Research and Practice
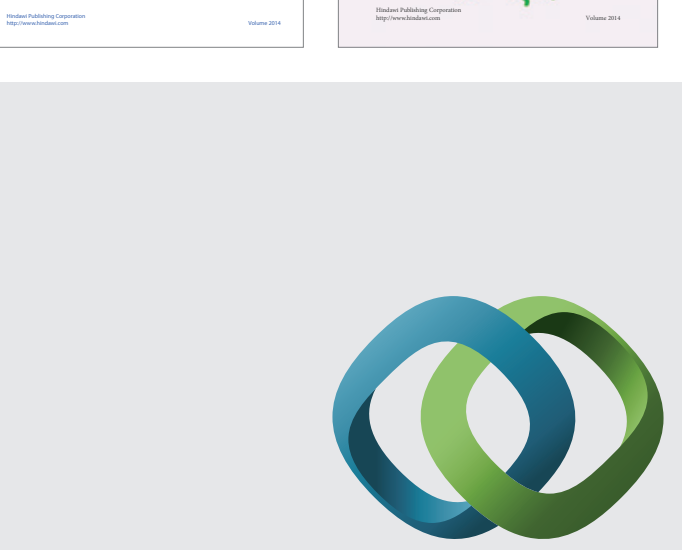

\section{Hindawi}

Submit your manuscripts at

http://www.hindawi.com
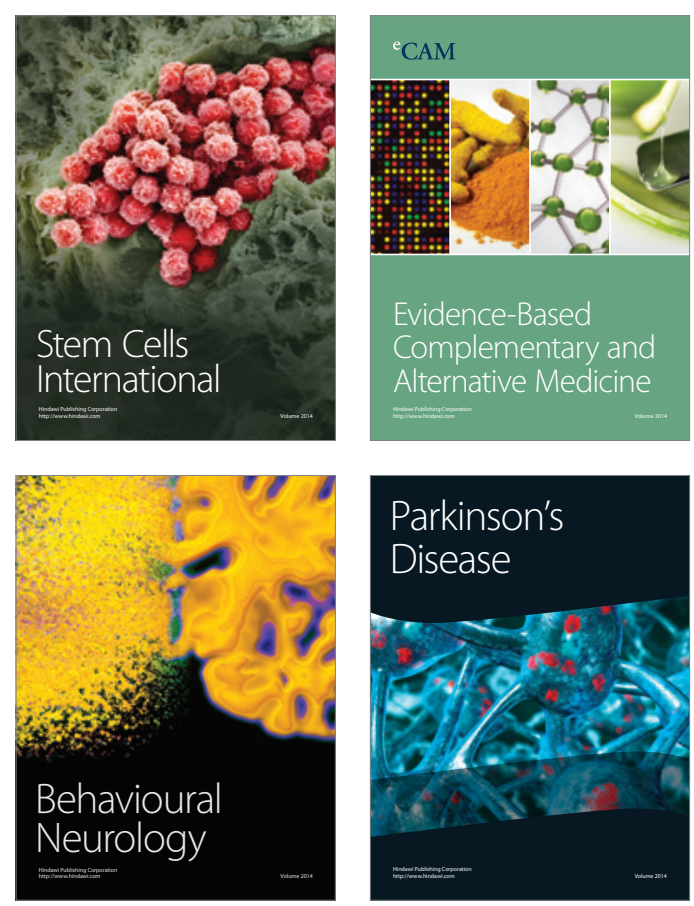

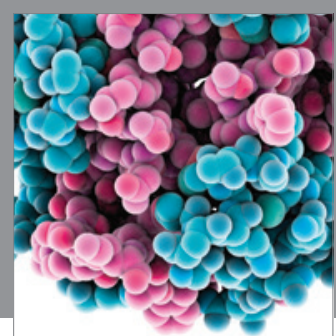

Journal of
Diabetes Research

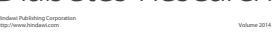

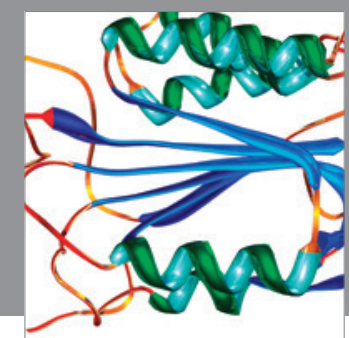

Disease Markers
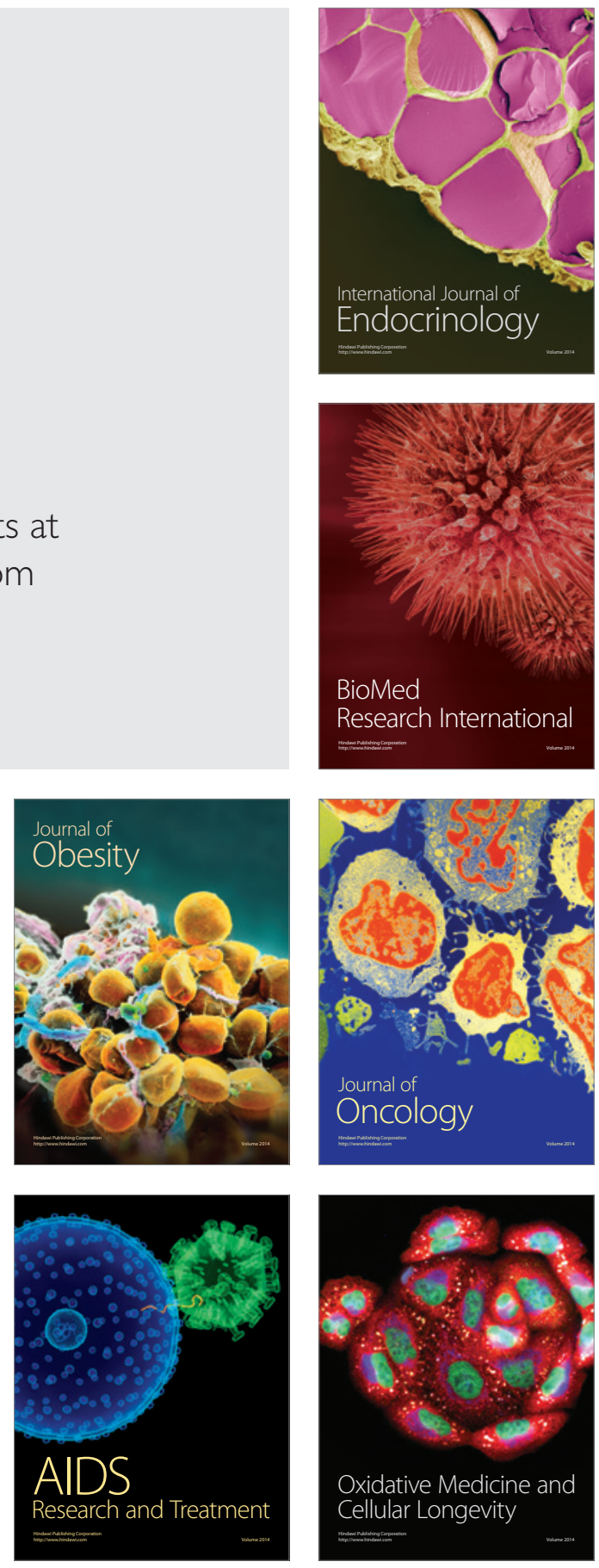